\title{
Entrustable Professional Activities in der psychiatrischen Weiterbildung: Ein vielversprechendes Konzept
}

\author{
Entrustable Professional Activities in Graduate Medical \\ Education in Psychiatry: A Promising Concept
}

Severin Pinilla ${ }^{1,2, a}$, Oliver Matthes,3,a, Anouk Gehret ${ }^{12}$, Sören Huwendiek², Eric Lenouvel ${ }^{1}$, Susanne Lindert ${ }^{4}$, Adrian Philipp Marty ${ }^{5}$, Beat Nick ${ }^{6,13}$, Christoph Nissen ${ }^{7}$, Andres Schneeberger ${ }^{8}$, Lea Stocker ${ }^{9}$, Michael Wallies ${ }^{10}$, Steffi Weidt ${ }^{11}$, Anja Weiss-Breckwoldt ${ }^{12}$ und Stefan Klöppel ${ }^{1,13}$

\author{
Universitätsklinik für Alterspsychiatrie und Psychotherapie, Universität Bern \\ Institut für Medizinische Lehre (IML), Abteilung für Assessment und Evaluation, Universität Bern \\ Klinik für Konsiliarpsychiatrie und Psychosomatik, Universitätsspital Zürich \\ Clienia Schlössli, Privatklinik und Lehrkrankenhaus der Universität Zürich, Oetwil am See/Zürich \\ 5 Institut für Anästhesiologie, Universitätsspital Zürich \\ 6 Psychiatrische Dienste der Spitäler AG, Solothurn, Psychiatrische Dienste der Spitäler AG Solothurn, \\ Präsident der Prüfungskommission und Mitglied der Kommission Weiter- und Fortbildung (SKWF), \\ Schweizerische Gesellschaft für Psychiatrie und Psychotherapie (SGPP) \\ Universitätsklinik für Psychiatrie und Psychotherapie, Universität Bern \\ Psychiatrische Dienste Graubünden, Chur \\ ${ }^{9}$ Akuttagesklinik, Psychiatrische Universitätsklinik Zürich \\ ${ }^{10}$ Privatklinik für Psychiatrie und Psychotherapie, Clienia Littenheid \\ 'Psychiatrische Universitätsklinik Zürich \\ ${ }^{2}$ Ambulatorium Römerhof, Zürich \\ ${ }^{13}$ Ressort Qualität der Ständigen Kommission Weiter- und Fortbildung (SKWF), Schweizerische Gesellschaft \\ für Psychiatrie und Psychotherapie (SGPP) \\ a Geteilte Erstautorenschaft
}

\begin{abstract}
Zusammenfassung: Entrustable Professional Activities (EPAs) oder anvertraubare professionelle Tätigkeiten (APTs) sind kompetenzbasierte Lernziele, die sich aus beobachtbaren klinischen Tätigkeiten ableiten. Im Studium der Humanmedizin haben sie mittlerweile schweizweit als Teil des sogenannten PROFILES-Katalogs (Principal Relevant Objectives and Framework for Integrated Learning and Education in Switzerland) Einzug gehalten. Es ist naheliegend, in der Weiterbildung an die neun im Studium vermittelten EPAs anzuknüpfen und EPAs in der Weiterbildung einzuführen. Wir diskutieren diesen Ansatz für die Weiterbildung zum Facharzt Psychiatrie und Psychotherapie aus Sicht verschiedener Weiterbildungskontexte und eines Praxisbeispiels. In diesem Positionspapier beschreiben wir eine Chance für Verbesserung durch die Implementierung von EPAs sowohl in Bezug auf die Qualität der ärztlichen Weiterbildung und damit der Patient_innenversorgung als auch in Bezug auf die Attraktivität des Fachgebiets für ärztlichen Nachwuchs.
\end{abstract}

Schlüsselwörter: EPAs, kompetenzbasierte Weiterbildung, Psychiatrie

Abstract: Entrustable Professional Activities (EPAs) are competency-based learning goals derived from observable clinical activities. In undergraduate medical education, they have now been adopted throughout Switzerland as part of the so-called PROFILES catalog (Principal Relevant Objectives and Framework for Integrated Learning and Education in Switzerland). The nine core EPAs to be mastered in undergraduate medical education can serve as a basis for introducing EPAs in graduate medical education as well. We will discuss this approach in the context of graduate medical education in psychiatry and psychotherapy from the perspective of different training contexts and a pilot example. In this position paper, we describe a promising opportunity to improve graduate medical training through the implementation of EPAs, both in terms of the quality of training and thus of patient care, as well as in terms of the attractiveness of the specialty for future residents.

Keywords: EPAs, competency-based medical education, psychiatry 
Résumé: Les activités professionnelles pouvant être réalisés de manière autonome (EPA) ou les activités professionnelles déléguées (APDs) sont des objectifs d'apprentissage basés sur les compétences dérivées d'activités cliniques observables. Dans l'étude de la médecine humaine, ont maintenant été introduites dans toute la Suisse dans le cadre du catalogue dit PROFILES (Principal Relevant Objectives and Framework for Integrated Learning and Education in Switzerland). Il est évident que les neuf EPAs enseignées au cours des études devraient servir de base à la formation continue et que les EPAs devraient être introduites dans la formation continue. Nous aborderons cette approche de la formation continue en psychiatrie et en psychothérapie sous l'angle de divers contextes de formation continue et d'un exemple pratique. Dans cette exposé de position, nous résumons des chances d'amélioration grâce à la mise en œuvre des EPA, à la fois en termes de qualité de la formation médicale continue et donc des soins aux patients, ainsi qu'en termes d'attrait du domaine pour les jeunes médecins.

Mots-clés: EPAs, éducation basée sur des compétences, psychiatrie

\section{Hintergrund}

Entrustable Professional Activities (EPA) oder anvertraubare professionelle Tätigkeiten (APT) sind kompetenzbasierte Lernziele, die sich aus beobachtbaren klinischen Tätigkeiten ableiten. Als solche sind sie Kernelemente im Kontext von Kompetenz-basierten Aus- und Weiterbildungscurricula in der Medizin [1, 2]. Hinter der Ausrichtung auf Kompetenz als übergeordnetes Bildungsziel steht die stärkere Ergebnisorientierung in der Ausbildung (im Medizinstudium) und der Weiterbildung (während der Facharztweiterbildung). Weitere Ziele sind, individuelle Aus- und Weiterbildungswege (Kompetenz- versus Zeitabhängig) zu ermöglichen und die kontinuierliche Verbesserung der Patientensicherheit [3].

Mittlerweile haben Lehrverantwortliche in vielen Ländern und Fachgebieten (und Gesundheitsberufen allgemein) sowohl auf der Ausbildungsebene als auch auf der Weiterbildungsebene die Entwicklung, Implementierung und Überprüfung von Kompetenz-basierten Curricula mit professionellen Rollen (typischerweise den CanMEDSRollen) und EPAs begonnen [4-8]. Die CanMEDS-Rollen bilden bereits jetzt die Grundlage für den allgemeinen Lernzielkatalog der schweizerischen Weiterbildungsprogramme [9]. EPAs reflektieren hier vor allem die klinische Aus- und Weiterbildung tendenziell besser als traditionelle Kataloge, da sie Lernziele in Bezug auf Wissen, Fähigkeiten, Fertigkeiten und Haltungen aus Sicht einer klinischen Tätigkeit vereinen. Die internationalen Qualitätskriterien für die EPA-Entwicklung sind in Tabelle 1 aufgeführt.

Im Rahmen einer Implementierungsstudie aus Deutschland wurden EPAs bereits in der klinisch-praktischen Ausbildung von Medizinstudierenden im Fach Psychiatrie eingeführt [10]. Im schweizerischen Kontext wurde ein nationaler EPA-basierter Lernzielkatalog für die Ausbildung bereits veröffentlicht [11]. Die dort definierten neun Kern-EPAs (Tabelle 2) sollen während der Ausbildung erlernt werden, sodass Medizinstudierende am ersten Tag ihrer Weiterbildung (unabhängig vom Fachgebiet) die Tätigkeiten der neun Kern-EPAs sicher unter indirekter Supervision ausführen können. Erste Pilotprojekte in der klinisch-praktischen Ausbildung von Medizinstudierenden im Fach Psychiatrie an der Universität Bern wurden positiv evaluiert [12].
Im Gegensatz zur Ausbildung stehen die Weiterbildungscurricula noch am Anfang der Einführung von EPAs. Im Rahmen lokaler Projekte auf der Ebene einzelner medizinischer Fakultäten und Weiterbildungsstätten, unter anderem im Fach Psychiatrie, wurden EPA-basierte Curricula entwickelt. In der Schweiz haben auch verschiedene Fachgesellschaften, zum Beispiel die chirurgische und internistische, auf nationaler Ebene begonnen, an die in der Ausbildung erworbenen EPAs anzuknüpfen und Kompetenzprofile der jeweiligen Fachgebiete abzubilden.

Auch international zeigt sich dieser Trend in einigen Fachgebieten (z.B. Kardiologie, Anästhesie, Pädiatrie). Typischerweise werden EPAs in einem mehrstufigen Verfahren entwickelt und validiert [13]. Für das Fachgebiet Psychiatrie und Psychotherapie konnten im Rahmen einer Übersichtsarbeit 20 Artikel identifiziert werden, die in unterschiedlicher Gewichtung auf die Entwicklung, Implementierung und Überprüfung von EPAs fokussieren [14]. Länder wie Australien und Neuseeland haben bereits das gesamte psychiatrische Weiterbildungscurriculum mit Hilfe von EPAs strukturiert. In den letzten Jahren wurden EPAs für verschiedene psychiatrische Weiterbildungsstufen und -schwerpunkte ebenfalls in den USA [15] und Kanada [16] entwickelt. Die valide und reliable Überprüfung, ob eine EPA oder die Summe der EPAs kompetent beherrscht wird, ist eine komplexe Aufgabe und aktuell Gegenstand verschiedener medizindidaktischer Forschungsprojekte. Theoretisch bieten EPA-basierte Curricula grundsätzlich sowohl die Möglichkeit, bessere formative Assessments (im Sinne von «Low Stakes», Unterstützung des Lernprozesses von Weiterzubildenden) als auch summative Kompetenzentscheidungen (im Sinne von «High Stakes», z.B. Ist ein/e Kandidat/in bereit für Nachtdienste? Ist ein/e Kandidat/in bereit für die selbständige Tätigkeit in einer Praxis?) zu treffen.

In der Literatur werden meist Entrustment-Supervision-Skalen beschrieben [17], um im klinischen Alltag einzuschätzen, wie selbständig Weiterzubildende eine umschriebene Tätigkeit (entsprechend einer EPA) ausführen

\footnotetext{
Im Artikel verwendete Abkürzungen

APT Anvertraubare professionelle Tätigkeiten

EPA Entrustable Professional Activities

SGPP Schweizerische Gesellschaft für Psychiatrie und Psychotherapie
} 
können. Diese Einschätzung der Supervisionsnotwendigkeit in Kombination mit Feedback für eine bestimmte klinische Tätigkeit ist die zentrale Besonderheit des EPAAnsatzes. Aus den jeweiligen formativen arbeitsplatzbasierten Assessments ergeben sich idealerweise direkte handlungsorientierte Rückmeldungen an die Weiterzubildenden, um die nächsthöhere Selbständigkeitsstufe zu erreichen. Entsprechende Supervisionsstufen basierend auf den Empfehlungen des SIWFs im Fachgebiet Psychiatrie sind in Abbildung 1 dargestellt.

Für die erfolgreiche Implementierung von EPA-basierten Weiterbildungscurricula ist die Feedback-Qualität im Rahmen der arbeitsplatzbasierten Assessments (z.B. MiniClinical Evaluation Excercise (MiniCEX), eine Kurzbeobachtung durch den/die supervidierende/n Oberarzt/ärztin mit anschliessendem Feedbackgespräch unter Verwendung einer Entrustment-Supervisionsskala [17]) entscheidend. Neben dieser Form kommen zahlreiche weitere Arbeitsplatz-basierte Assessments (u.a. klinische Fallvorstellungen, strukturierte Durchsicht einer Stichprobe der Krankengeschichtenführung, wissenschaftliche Präsentationen, Multi-Source-Feedback) infrage [18]. Zudem können weitere Assessmentformate für die Einschätzung der Supervisionsnotwendigkeit eingesetzt werden, wie simulationsbasierte Prüfungen (bei der Kombination mehrerer Prüfungssimulationen z.B. als Objective Structured Clinical Examination, OSCE) [19] oder auch Prüfungen bezüglich des Anwendungswissens [20].

Für summative Prüfungsentscheidungen werden entsprechende Prüfungsprogramme («Programmatic Assessment») empfohlen [21]. Im Sinne von Programmatic Assessment soll sich auch die Prüfungskultur ändern und die Reihenfolge von Lernen und Prüfen flexibler gestaltet werden. Anstatt zu versuchen, jemandem etwas beizubringen und am Schluss zu prüfen, ob es beherrscht wird («Assessment of Learning»), wird zuerst eine «Assessment-Diagnostik» durchgeführt und anschliessend geplant, was gelernt werden soll («Assessment for Learning»). Ein weiterer Aspekt dieser Prüfungsprogramme ist, dass möglichst viele Bewertungspunkte pro Kandidat/ in gesammelt werden (Abb. 2), bei den einzelnen Bewertungspunkten Feedback an die Weiterzubildenden gegeben wird und die «High Stakes»-Entscheidungen (z.B. die Vergabe des fachärztlichen Titels) im Rahmen eines Gremiums («Clinical Competence Committee») auf Basis der gesammelten Datenpunkte getroffen werden. Ein solches Prüfungsprogramm zielt primär darauf $a b$, Lernprozesse zu unterstützen, kann aber auch effektiver genutzt werden, um ein «Durchwinken» von Kandidat/innen begründet zu vermeiden. Zur Unterstützung der Umsetzung eines solchen Prüfungsprogramms gerade über mehrere Weiterbildungsstätten hinweg bieten sich digitale Lösungen wie Prüfungs-Apps [22] oder e-Portfolios an [23].

\section{Praxisbeispiel am Universitätsspital Zürich}

In der Klinik für Konsiliarpsychiatrie und Psychosomatik am Universitätsspital Zürich (USZ) beginnt derzeit ein Pilotprojekt, in dem die Einarbeitung und Weiterbildung in den Bereich der Notfall- und Konsiliarpsychiatrie über sieben EPAs erfolgen soll. Eine Besonderheit ist dabei, dass die arbeitsplatzbasierten Assessments mit Hilfe der «prEPAred»-App [22] durchgeführt werden. Dies redu-

Titel

Abklärung der Urteilsfähigkeit

Beschreibung

Die EPA umfasst den gesamten Konsilauftrag inklusive Entgegennehmen der Konsilanfrage, vorbereitendes Aktenstudium, allfälliges Vorbesprechen mit den Behandlungsteams, Exploration der*s Patienten*in, allfällige Exploration weiterer involvierter Personen (beispielsweise Fremdanamnese bei Angehörigen), Vorstellung des Falls bei dem*r zuständigen Oberärzt*in, Dokumentation sowie Leistungserfassung, allfällige Rückmeldungen an das Behandlungsteam und ggf. Übergabe des Falles.

Wissen, Fähigkeiten, Fertigkeiten und Haltungen

- Wissen: Kenntnisse der relevanten Dokumente des Notfallordners, der USZ-internen Weisungen sowie der rechtlichen und ethischen Grundlagen.

- Fähigkeiten/Fertigkeiten: Anamnese- und Befunderhebung, spezifische Beurteilung der Frage nach Urteilsfähigkeit ggf. unter Einbezug strukturierter Fragebögen (U-Doc), konzise Dokumentation und Fallvorstellung bei Oberärzt*in, ggf. Patientenübergabe.

- Haltungen: Kollegiale Haltung gegenüber dem Behandlungsteam, respektvolle und professionelle Haltung gegenüber der*m Patienten*in, eigene Grenzen kennen.
Mögliche Risiken

Bei nicht sachgerechter Beurteilung können Autonomie- und Selbstbestimmungsrechte von Patient*innen verletzt werden und ungerechtfertigte medizinische Behandlungen zur Folge haben.

Essentielle Kompetenzdomänen

Medical Expert (Medizinische*r Expert*in)

Communicator (Kommunikator*in)

Collaborator (Mitglied eines Teams)

\section{Assessment/Evaluation}

Insbesondere: Narratives Feedback in der prEPAred-App Auch möglich: Wissenstest (mündlich oder schriftlich), Mini-CEX, Case-based Discussion, Multi-Source-Feedback (Patient*in/ Angehörige/fallführendes Behandlungsteam), Simulation mit Schauspielpatient*in

Angestrebtes Niveau

Mindestens Supervisionslevel 3 = Grundlage für Erreichen der Dienstfähigkeit (Assessment durch für Einarbeitung zuständiger*n Oberärzt*in)

Abbildung 1. Beispiel der Definition einer EPA (gekürzt, basierend auf Leitlinie zur Implementierung von Entrustable Professional Activities (EPAs) in die Weiterbildungsprogramme, SIWF 28.11.2019). 


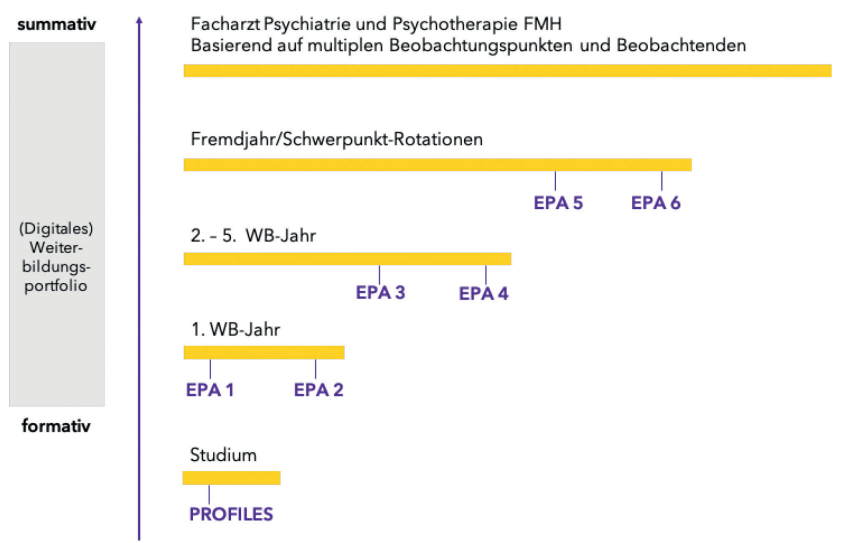

Abbildung 2. Schematische Übersicht: Integriertes EPA-basiertes Aus- und Weiterbildungscurriculum. Für das Weiterbildungscurriculum sollten EPAs nach Weiterbildungsrotation (z.B. alterspsychiatrische und suchtmedizinische Rotationen) und Kontext (z.B. Basisjahr, stationäres und ambulantes Kerncurriculum) definiert werden. Die longitudinale Abbildung in einem e-Portfolio erlaubt eine individualisierte Lernprozessunterstützung über einzelne Weiterbildungsstätten hinweg. Für jede EPA wird festgelegt, welche Supervisionsstufe erreicht sein muss, um fachärztlich tätig sein zu dürfen.

ziert im Vergleich zum Mini-CEX als Assessmentinstrument den Dokumentationsaufwand, soll das Feedback niederschwelliger machen und den persönlichen Austausch zwischen Supervidierenden und Weiterzubildenden stärken. Die App wird bereits in der Anästhesiologie angewandt und gegenwärtig auch im Fachbereich Chirurgie implementiert und evaluiert. Die Weiterzubildenden erstellen damit über die Weiterbildungszeit ein digitales Lernportfolio über die erworbenen Kompetenzen und die erhaltenen Feedbacks im Bereich der Notfall- und Konsiliarpsychiatrie.

Die Liste der EPAs (Tabelle 3) hat nicht den Anspruch, die gesamte Tätigkeit abzubilden. Sie wurden vielmehr aus Sicht der Weiterzubildenden im Vordergrunddienst erstellt und sollen insbesondere diejenigen beobachtbaren klinischen Arbeitssituationen abbilden, die regelhaft als besonders herausfordernd erlebt werden. Am Beispiel der

Tabelle 1. Kriterien für die EPA-Entwicklung und Definition (nach [34])

Abgrenzbare Tätigkeit

Klarer Beginn und klares Ende

Essenziell und relevant für das Fachgebiet

Delegierbar

Führt zu einem klinisch relevanten Ergebnis

Messbares Ergebnis

Nur durch qualifiziertes Personal durchführbar

Beobachtbar
EPA «Abklärung von Urteilsfähigkeit» soll der Aufbau einer EPA-Beschreibung erläutert werden (Abb. 1). Als Mass für die Kompetenz in einer umschriebenen klinischen Arbeitssituation wird das Level an benötigter Supervision auf einer Skala abgebildet (Tabelle 4). In der App wird neben der Selbsteinschätzung des Trainees die Fremdeinschätzung durch die Supervisorin/den Supervisor festgehalten.

Um zu überprüfen, ob die Einführung von EPAs die Weiterbildungsqualität steigert, wird die Einführung wissenschaftlich im Sinne eines «Proof-of-Concept»-Pilotprojektes evaluiert. Primär wird anhand von qualitativen Daten untersucht, ob eine App als Feedbackinstrument im klinischen konsiliarpsychiatrischen Setting geeignet ist. Mittels einer Befragung der Weiterzubildenden sollen auch quantitative Indikatoren bezüglich der Nutzung und Anwendung ausgewertet werden.

\section{Ausblick}

Ein kompetenzbasiertes Curriculum für die psychiatrische Weiterbildung sollte die Kern-EPAs der Ausbildungsebene (relevant für alle Medizinstudierenden, unabhängig von der späteren Weiterbildung) für die EPAs der fachspezifischen Weiterbildung berücksichtigen. In Sounding Boards der Schweizerischen Gesellschaft für Psychiatrie und Psychotherapie (SGPP) zur Weiter- und Fortbildung (zuletzt im Juni 2020) zur Diskussion der aktuellen Weiterbildungsordnung wird deutlich, dass die Erwartung an die zu vermittelnden Kompetenzen steigen, gleichzeitig aber weder die Dauer der Weiterbildung noch deren Komplexität (z.B. durch zusätzliche Pflichtrotationen) erhöht werden soll. Eine stärkere Kompetenz-Orientierung durch den Einsatz von EPAs kann grosses Potenzial für die klinische Weiterbildung und letztlich die Patientenversorgung entwickeln. Im Fach Psychiatrie und Psychotherapie besteht aus unserer Sicht eine besondere Notwendigkeit einer Veränderung hin zu einem kompetenzbasierten Curriculum: Es besteht eine grosse Diskrepanz zwischen der minimalen (sechs Jahre) [24] und der durchschnittlichen (neun

Tabelle 2. Kern-EPAs (nach [11]) im Medizinstudium

Eine Anamnese erheben

Den Somato- und Psychostatus erheben

Eine differenzialdiagnostische Einordnung vornehmen

Diagnostische Untersuchungen verordnen

Allgemeine ärztliche Tätigkeiten (wie z.B. Blutentnahme)

Einen Notfall initial versorgen

Einen Medikations- und Behandlungsplan erstellen

Die ärztliche Dokumentation durchführen und das Vorstellen von Patient/innen

Die Sicherheitskultur mittragen (z.B. einen Critical-Incident-Report erstellen) 
Jahre) [25] Weiterbildungszeit. Eine mögliche Erklärung könnte ein höherer Anteil von Teilzeitbeschäftigten sein. Empirische Daten liegen unseres Wissens zu dieser gesamtgesellschaftlich relevanten Thematik und in Bezug auf die Verschiebung der Geschlechterverhältnisse im Medizinstudium und den ärztlichen Berufen leider nicht vor. EPAs könnten zumindest helfen, das Erreichen von notwendigen Kompetenzen unabhängig von reinen Zeitfaktoren zu operationalisieren und flexiblere Weiterbildungswege zu ermöglichen. Eine amerikanische Studie zu EPAs im Kontext der Pädiatrie lieferte Hinweise darauf, dass eine Individualisierung der Aus- und Weiterbildungszeiten abhängig vom Erreichen der Kompetenzziele möglich ist [26].

Durch transparente, EPA-basierte Kompetenzprofile als Basis für individualisiertes Teaching kann auch eine Effizienzsteigerung der Weiterbildung erwartet werden. Hier müssen gegebenenfalls auch zusätzliche Ressourcen für die Lehrkompetenzentwicklung bei Kaderpersonal («Teaching to Teach») eingesetzt werden. Zugleich könnte die Psychiatrie von innovativen Ansätzen in der Weiterbildung profitieren, um ärztlichen Nachwuchs für das Fachgebiet zu motivieren [27]. Aus Sicht der Weiterbildungsassistent/innen könnten Lernziele in Form von EPAs (im Sinne einer «mastery-approach»-Haltung) auch günstiger zur Förderung selbstregulierten Lernens am Arbeitsplatz sein [28]. Dies hängt jedoch von der Art des Einsatzes Arbeitsplatzbasierter Assessments ab. Spannungsfelder entstehen bei Standardisierung versus Individualisierung von Lernzielen, bei Performanzüberprüfung versus Lernunterstützung und bei Skalenbewertung versus narratives Feedback und Dialog [29]. Zur vollständigen Ausarbeitung einer EPA gehört auch eine zumindest grobkursorische Beschreibung des relevanten Wissens, der Fähigkeiten, Fertigkeiten und der Haltungen [30]. Das SIWF bietet hierfür zahlreiche Ressourcen an [31]. Bei der Implementierung sind zudem die Einbindung und Weiterbildung der Beteiligten hinsichtlich dieses Ansatzes zentral. Eine erste Anwendungsstudie aus den USA zeigte positive Ergebnisse für digitalisierte Arbeitsplatz-basierte Assessments von EPAs in der psychiatrischen Weiterbildung mittels einer App [32].

Eine Herausforderung für die Entwicklung eines Kompetenz-basierten psychiatrischen Weiterbildungscurriculums basierend auf EPAs wird die Integration psychiatrischer und psychotherapeutischer Lern- und Entwicklungsziele sein, die die Identität des Faches Psychiatrie mitprägen: Hierzu zählen beispielsweise ein mehrdimensionales Verständnis von psychischer Gesundheit im Spannungsfeld von Neuro- und Sozialwissenschaften, das differenzierte Erfassen und Gewichten des bio-psycho-sozialen Ansatzes in der psychiatrischen Arbeitsweise und die bewusste Auseinandersetzung mit der eigenen Persönlichkeitsentwicklung [33]. In Hinblick auf die Nachwuchsrekrutierung erscheint es angesichts der häufig frühen Entscheidung von Medizinstudierenden für ein Fachgebiet sinnvoll, die Grundlagen dieser Kompetenz-basierten Lernziele möglichst auch schon im vorklinischen Bereich zu verankern.
Tabelle 3. Liste der EPAs der Notfall- und Konsiliarpsychiatrie USZ

Management einer/s agitierten Patienten/in

Management einer/s Haftpatienten/in

Management einer/s suizidalen Patienten/in

Management einer/s deliranten Patienten/in

Management einer/s Suchtpatienten/in

Dringende Transplantations-Abklärung

Abklärung von Urteilsfähigkeit

Im Hinblick auf die konkrete Umsetzung und einer EPAbasierten Weiterbildung in der Psychiatrie können wir auf die Vorarbeiten aus anderen Ländern bzw. Fachbereichen zurückgreifen. Wenngleich international Vorbilder existieren, sollten diese für den spezifischen schweizerischen Kontext - idealerweise in einem nationalen Delphi-Verfahren (im Sinne einer systematischen und mehrstufigen Befragung) und unter Berücksichtigung der Sprachregionen validiert werden. Dies würde den Aufwand für die einzelne Weiterbildungsstätte deutlich reduzieren. Der vor allem initial zu erwartende hohe Aufwand für die EPA-Entwicklung und Auswahl von Arbeitsplatz-basierten Prüfungsmethoden und -vorgehen kann und sollte auf mehrere Standorte in verschiedenen Sprachregionen verteilt werden. Während für die Alterspsychiatrie eine Pflichtrotation besteht, müssen Kompetenzen und Fertigkeiten bei der Diagnostik und Behandlung z.B. von Suchterkrankungen oft ohne Rotation in eventuell vorhandene spezialisierte Bereiche erfolgen. Besondere Herausforderungen stellen sich aber auch in Bereichen wie der Konsiliar- und Liaisonpsychiatrie, da dort die direkte Supervision organisatorisch oft schwieriger ist. Hier sollten sich die Schwerpunktgesellschaften einbringen und Empfehlungen für angemessenes Arbeitsplatz-basiertes Assessment formulieren.

In der Zusammenschau sehen wir für das Fachgebiet Psychiatrie in der stärkeren Kompetenzorientierung durch die Implementierung von EPAs eine gute Chance für Verbesserung sowohl in Bezug auf die Qualität der ärztlichen Weiterbildung und damit der Patient_innenversorgung als auch in Bezug auf die Attraktivität des Fachgebiets für ärztlichen Nachwuchs. Konkrete nächste Schritte für eine

Tabelle 4. Supervisionslevel für EPAs

Level 1: Weiterbildungskandidat/in darf zuschauen Level 2 (direkte Supervision): Supervisor/in ist durchgehend vor Ort

Level 3 (reaktive (indirekte) Supervision): Supervisor/in ist bei Bedarf schnell vor Ort - telefonische Supervision durch Hintergrund-Oberärzt/in in der Regel zur Ausübung der EPA ausreichend, ggf. punktuell direkte Supervision notwendig

Level 4 (entfernte Supervision): Supervision findet retrospektiv statt - im Wesentlichen selbständige Ausübung der EPA, Nachbesprechung mit zuständiger/m Oberärzt/in in der Regel ausreichend

Level 5: Darf andere supervidieren 
stärkere Kompetenzorientierung der psychiatrischen Weiterbildung wären eine systematische Nutzung internationaler Vorarbeit, Pilotierungsprojekte in lokalen Kontexten mit medizindidaktischer Begleitforschung und eine nationale Validierung über die SGPP.

\section{Bibliografie}

1. Frick S. EPAs, Milestones, Competences: Was brauchen wir davon? Bulletin des médecins suisses. 2019;100(8):260-263.

2. ten Cate O. Entrustability of professional activities and competency-based training. Med Educ. 2005;39(12):1176-1177

3. Hodges B, Lingard L (editors). The question of competence: reconsidering medical education in the twenty-first century. The Culture and Politics of Health Care Work. New York; Cornell University Press: 2012

4. O'Dowd E, Lydon S, O'Connor P, Madden C, Byrne D. A systematic review of 7 years of research on entrustable professional activities in graduate medical education, 2011-2018. Med Educ. 2019;53(3):234-249.

5. Meyer EG, Chen HC, Uijtdehaage S, Durning SJ, Maggio LA. Scoping review of Entrustable Professional Activities in undergraduate medical education. Acad Med. 2019;94(7):1040-1049.

6. Shorey S, Lau TC, Lau ST, Ang E. Entrustable professional activities in health care education: a scoping review. Med Educ. 2019;53(8):76677.

7. Holmboe ES, Sherbino J, Englander R, Snell L, Frank JR; ICBME Collaborators. A call to action: The controversy of and rationale for competency-based medical education. Med Teach. 2017;39(6):574-581.

8. Sottas B. Learning outcomes for health professions: the concept of the swiss competencies framework. GMS Z Med Ausbild. 2011;28(1):Doc11.

9. Schweizerisches Institut für ärztliche Weiter- und Fortbildung (SIWF). Lernzielkatalog 2020 [cited 2020 June 2]. https://www. siwf.ch/files/pdf17/allg_lz_d.pdf; letzter Zugriff: 01.10.2020.

10. Koelkebeck K, Brouwer B, Ahrens H Becker JC. Einführung und Evaluation eines neuen Kurrikulums Psychiatrie und Psychotherapie [Implementation and evaluation of a revised curriculum for psychiatry and psychotherapy]. Nervenarzt. 2019;90(11):1170-1176

11. Michaud PA, Jucker-Kupper P, Profiles Working Group. PROFILES; Principal Relevant Objectives and Framework for Integrated Learning and Education in Switzerland. Bern; Joint Commission of the Swiss Medical Schools: 2017.

12. Pinilla S, Cantisani A, Klöppel S, Strik W, Nissen C, Huwendiek S. Introducing a clerkship curriculum based on entrustable professional activities: a pilot study. Manuscript submitted for publication, 2020. DOI: 10.21203/rs.3.rs-25193/v1

13. Kwan J, Crampton R, Mogensen LL, Weaver R, van der Vleuten CPM, Hu WCY. Bridging the gap: a five stage approach for developing specialty-specific entrustable professional activities. BMC Med Educ. 2016;16:117.

14. Pinilla S, Lenouvel E, Strik W, Klöppel S, Nissen C, Huwendiek S. Entrustable Professional Activities in psychiatry: A systematic review. Acad Psychiatry. 2020;44(1):37-45.

15. Young JQ, Hasser C, Hung EK, et al. Developing End-of-Training Entrustable Professional Activities for psychiatry: Results and methodological lessons. Acad Med. 2018;93(7):1048-1054.

16. Bryden P, Chatterjee S, Chaukos D, et al. Competency by Design. Handbook for the Department of Psychiatry [cited 2020 May ]. https://www.psychiatry.utoronto.ca/sites/default/files/ competency_by_design_handbook_-_20200721.pdf; letzter Zugriff: 01.10.2020.

17. ten Cate O, Schwartz A, Chen HC. Assessing trainees and making entrustment decisions: On the nature and use of entrustment-supervision scales [published online ahead of print, 2020 Apr 21]. Acad Med. 2020;10.1097/ACM.0000000000003427.

\section{Key messages}

- Entrustable Professional Activities bieten sich für eine kompetenz-basierte Weiterbildungsreform im Fachgebiet Psychiatrie und Psychotherapie an.

- In einem ersten Schritt müssen fachspezifische EPAs für die jeweiligen Weiterbildungsabschnitte und mit entsprechenden arbeitsplatz-basierten Assessments entwickelt werden.

- EPA-basierte Weiterbildungscurricula ermöglichen individualisierte Weiterbildungscurricula, können selbst-reguliertes Lernen am Arbeitsplatz unterstützen und dienen letztlich der Verbesserung der Patientinnenversorgung.

18. Norcini J, Burch V. Workplace-based assessment as an educational tool: AMEE Guide No. 31. Med Teach. 2007;29(9):855-71.

19. Hodges BD, Hollenberg E, McNaughton N, Hanson MD, Regehr G. The Psychiatry OSCE: a 20-year retrospective. Acad Psychiatry. 2014;38(1):26-34.

20. Lloyd RB, Park YS, Tekian A, Marvin R. Understanding assessment systems for clinical competency committee decisions: Evidence from a multisite study of psychiatry residency training programs. Acad Psychiatry. 2019;10.1007/s40596-019-01168-x.

21. van der Vleuten CP, Schuwirth LW. Assessing professional competence: from methods to programmes. Med Educ. 2005:39(3):309-317.

22. Marty A. prEPAred - Assessment App [computer software]. Labs PTE LTD; 2020. https://www.apple.com/ios/app-store/; letzter Zugriff: 01.10.2020.

23. de Swardt M, Jenkins LS, von Pressentin KB, Mash RM. Implementing and evaluating an e-portfolio for postgraduate family medicine training in the Western Cape, South Africa. BMC Med Educ. 2019;19(1):251.

24. Schweizerisches Institut für ärztliche Weiter- und Fortbildung (SIWF). Weiterbildungsprogramm - Facharzt für Psychiatrie und Psychotherapie. Geschäftsbericht Schweizerisches Institut für ärztliche Weiter- und Fortbildung SIWF 2020 [cited 2020 June 2nd, 2020]. https://www.siwf.ch/files/pdf7/psychiatrie_ version_internet_d.pdf; letzter Zugriff: 01.10.2020.

25. Schweizerisches Institut für ärztliche Weiter- und Fortbildung (SIWF). Fortbildung - Kompetenzen erhalten und weiterentwickeln. Geschäftsbericht Schweizerisches Institut für ärztliche Weiter- und Fortbildung SIWF 2020 [cited 2020 June 2nd, 2020]. https://www.siwf.ch/files/pdf23/geschaeftsberichtsiwf-2019.pdf; letzter Zugriff: 01.10.2020.

26. Murray KE, Lane JL, Carraccio C, et al. Crossing the gap: Using competency-based assessment to determine whether learners are ready for the undergraduate-to-graduate transition. Acad Med. 2019;94(3):338-345

27. Schneeberger A, Gupta R, Heuss S. Gamification - ein neuer Lernansatz in der Psychiatrie? Leading Opinions. Neurologie Psychiatrie. 2020;1:25-27

28. Daniels L, Daniels V. Internal medicine residents' achievement goals and efficacy, emotions, and assessments. Can Med Educ J. 2018;9(4):e59.

29. Martin L, Sibbald M, Brandt Vegas D, Russell D, Govaerts M. The impact of entrustment assessments on feedback and learning: Trainee perspectives. Med Educ. 2020;54(4):328236

30. ten Cate $O$, Young JQ. The patient handover as an entrustable professional activity: adding meaning in teaching and practice. BMJ Qual Saf. 2012:21 Suppl 1:i9-12.

31. Schweizerisches Institut für ärztliche Weiter- und Fortbildung (SIWF). Entrustable Professional Activities (EPA's) 2020 [cited 2020 June 22]. https://www.siwf.ch/siwf-projekte/epa.cfm; letzter Zugriff: 01.10.2020. 
32. Young JQ, McClure M. Fast, easy, and good: Assessing Entrustable Professional Activities in psychiatry residents with a mobile app. Acad Med. 2020. https://doi.org/10.1097/ACM.000000000 0003390

33. Guerrero APS, Beresin EV, Balon R, et al. The Competency Movement in psychiatric education. Acad Psychiatry. 2017;41(3):312-314.

34. Taylor DR, Park YS, Egan R, et al. EQual, a Novel Rubric to Evaluate Entrustable Professional Activities for Quality and Structure. Acad Med. 2017 Nov;92(11S Association of American Medical Colleges Learn Serve Lead: Proceedings of the 56th Annual Research in Medical Education Sessions):S110-S117.

\section{Historie}

Manuskript eingereicht: 10.07.2020

Nach Revision angenommen: 16.09.2020

\section{Interessenskonflikt}

Die Autorinnen und Autoren erklären, dass keine Interessenskonflikte bestehen.

\section{Danksagung}

Wir möchten uns herzlich für die hilfreichen und konstruktiven Reviewkommentare (anonym) bedanken. Ausserdem möchten wir Sebastian Euler danken, der das EPA-Projekt am Universitätsspital Zürich als Mentor betreut.

\section{Prof. Dr. med. Stefan Klöppel}

Ärztlicher Direktor UPD

Direktor und Chefarzt Universitätsklinik für Alterspsychiatrie und Psychotherapie

Murtenstrasse 21

3008 Bern

stefan.kloeppel@upd.ch 